\title{
Dual task-related gait changes in patients with mild cognitive impairment
}

\author{
Alberto Nascimbeni, MDa \\ Shiva Caruso, $\mathrm{MSc}^{\mathrm{b}}$ \\ Adriana Salatino, $\mathrm{PhD}^{\mathrm{b}}$ \\ Marinella Carenza, MSc $^{\mathrm{b}}$ \\ Marta Rigano, MDa \\ Andrea Raviolo, MSc ${ }^{\mathrm{a}}$ \\ Raffaella Ricci, $\mathrm{PhD}^{\mathrm{b}}$
}

\author{
${ }^{a}$ Rehabilitation Unit, S. Croce Hospital, Moncalieri, \\ Turin, Italy \\ ${ }^{\mathrm{b}}$ Department of Psychology, University of Turin, \\ Turin, Italy
}

Correspondence to: Alberto Nascimbeni

E-mail: alnascim@tin.it

\section{Summary}

Mild cognitive impairment $(\mathrm{MCl})$ entails a high risk of developing Alzheimer's dementia. In $\mathrm{MCl}$ patients gait impairment, which increases the risk of falls and institutionalization, is an early motor sign. A dualtask (DT) paradigm might improve the observation of this phenomenon.

The aim of this study was to investigate motor-cognitive interference in a sample of $\mathrm{MCl}$ patients and a group of matched healthy controls submitted to DT conditions. To this end, three different cognitive tasks were used: counting backwards, short story recall and a phonemic fluency task.

Overall, the patients, compared with the healthy participants, performed worse on the cognitive tasks and showed some degree of gait impairment. In the DT conditions, both groups showed significant gait disruption independently of the concomitant cognitive task. As regards cognitive performance, counting backwards worsened during dual tasking, while short story recall improved in both groups.

Overall, our results suggest that the use of a DT paradigm does not improve the early detection of $\mathrm{MCl}$. Our findings of enhanced story recall during walking might have interesting implications for rehabilitation of memory function.

KEY WORDS: dual task, gait analysis, mild cognitive impairment

\section{Introduction}

Mild cognitive impairment $(\mathrm{MCl})$ is a condition of cognitive decline, mostly involving memory and attention (Petersen and Negash, 2008; Levinoff et al., 2005). $\mathrm{MCl}$ does not significantly interfere with the ability to carry out normal activities of daily living and it differs from dementia, which is characterized by more severe and disabling cognitive decline (Gauthier et al., 2006). However, patients with $\mathrm{MCl}$ are at a high risk of developing Alzheimer's dementia (Aggarwal et al., 2006).

Besides cognitive decline, $\mathrm{MCl}$ patients may manifest early motor and gait dysfunction (Verghese et al., 2008; Eggermont et al., 2010). Gait impairment entails a high risk of falls and institutionalization, as has been shown both in healthy elderly individuals and in patients with dementia (Sheridan and Hausdorff, 2007). Moreover, motor dysfunction is associated with a high risk of developing dementia (Aggarwal et al., 2006).

The term cognitive-motor interference refers to the phenomenon in which the simultaneous performance of a cognitive and a motor task interferes with the performance of one or both of these tasks. In particular, carrying out a motor task, such as walking, while performing a demanding cognitive task may greatly interfere with the overall performance (Woollacot and Shumway-Cook, 2002). Studies on cognitive-motor interference, employing dual-task (DT) conditions, have demonstrated that limited attentional resources are needed for gait and postural control in healthy young adults (Beauchet et al., 2005a; Dubost et al., 2008), while the attentional load is greater in healthy elderly individuals (Plummer-D'Amato et al., 2011; Dubost et al., 2006) and in patients with neurological diseases, such as dementia (Allali et al., 2007). Dual tasking represents a cognitive challenge since it demands the allocation of attentional resources to concomitant tasks. Although significant DT interference has been demonstrated in dementia (Allali et al., 2007; Pettersson et al., 2007; Manckoundia et al., 2006), studies on the effects of DT conditions in $\mathrm{MCl}$ have not yielded unequivocal results. For instance, MonteroOdasso and colleagues (2009) observed worsened gait performances (lower walking speed and higher gait variability) during dual tasking in $\mathrm{MCl}$ patients, but they did not include a control group with which to compare these patients' performances. In studies that did employ a control group, some authors observed 
greater gait disruption in $\mathrm{MCl}$ patients than in healthy participants during dual tasking (Gillain et al., 2009; Montero-Odasso et al., 2012; Maquet et al., 2010; Muir et al., 2012), while others did not observe any difference between groups (Pettersson et al., 2007). For example, the latter authors demonstrated a lower walking speed in $\mathrm{MCl}$ patients with respect to controls but no differences in gait performance between the groups during the execution of a concomitant verbal fluency task. In this study, the effects of the DT condition on the cognitive task were not evaluated. Maquet et al. (2010) and Gillain et al. (2009), evaluating 20 seconds of stabilized walking using accelerometers, observed reduced stride frequency and walking speed during counting backwards in $\mathrm{MCl}$ patients with respect to healthy subjects. In these studies, impaired gait performance in patients with respect to controls was also observed during walking performed as a single task (Maquet et al., 2010; Gillain et al., 2009). On the other hand, Muir et al. (2012), using a six-meter electronic walkway, did not find any gait difference between $\mathrm{MCl}$ patients and healthy subjects during single tasking, but found significant gait disruption in the $\mathrm{MCl}$ patients during dual tasking, as shown by their decreased walking speed and increased gait variability. Counting backwards by sevens and naming animals caused more gait disruption than counting backwards by ones. Consistently, Montero-Odasso et al. (2012), using a six-meter walkway, found a greater effect of DT interference on gait performance in the $\mathrm{MCl}$ group during counting backwards by sevens than during a naming animals task. However, in the majority of these studies the effect of DT interference on the cognitive tasks was not evaluated. Thus, it is not possible to establish whether changes in motor performance were accompanied by changes in cognitive performance and vice versa. Unlike these studies, in the present study we employed a longer, although not fatiguing, recording time (60 seconds) for each condition. The use of a longer testing time yields better gait variability insight and allows reliable assessment of cognitive performance. To our knowledge, there are few studies that have investigated the possibility that different kinds of cognitive tasks may differentially affect gait performance in $\mathrm{MCl}$ (see, for example, Montero-Odasso et al., 2012; Muir et al., 2012) and none of them have assessed the effect of walking on the execution of the different cognitive tasks.

The aim of this study was to investigate motor-cognitive interference using three different cognitive tasks in a sample of $\mathrm{MCl}$ patients and a group of matched healthy controls. The results may help to clarify whether specific DT conditions may be useful for detecting early signs of dementia. Moreover, the evaluation of both motor and cognitive performances and the comparison with healthy individuals may provide useful insight into the possible preferential allocation of limited attentional resources in $\mathrm{MCl}$ patients carrying out a cognitive task during walking. The comparison of the effects of three different cognitive tasks may shed some light on optimal DT conditions for the early detection of cognitive decline in $\mathrm{MCl}$.

\section{Materials and methods}

\section{Participants}

Thirteen $\mathrm{MCl}$ patients and ten healthy controls participated in the study (Table I). For both groups the inclusion criteria were age (65-85 years) and the ability to walk independently. Subjects were excluded if they had central or peripheral neurological diseases (e.g. previous stroke, Parkinson's disease or polyneuropathies), musculoskeletal disorders such as arthrosis impairing posture or gait, recent acute illness or surgery, psychiatric disorders and/or the use of psychiatric drugs that may affect cognitive performance. $\mathrm{MCl}$ outpatients were recruited on the basis of a quantitative and qualitative diagnostic evaluation. Neuropsychological testing comprised the evaluation of: global cognitive function (Mini-Mental State Examination, MMSE) (Folstein et al., 1975); verbal (digit span) and visual (Corsi span test) short-term memory (Spinnler and Tognogni, 1987); long-term memory (short story recall task) (Spinnler and Tognogni, 1987); attention and visual search (the attentional matrices test) (Spinnler and Tognoni, 1987); executive functions (Frontal Assessment Battery, FAB) (lavarone et al., 2004); semantic and phonemic fluency (Spinnler and Tognoni, 1987); praxic abilities (bucco-facial and ideomotor apraxia tests) (Spinnler and Tognoni, 1987); functional status, i.e. activities of daily living (ADL) (Katz et al., 1963); and instrumental activities of daily living (Lawton and Brody, 1969). The patients were also assessed with the Milan Overall Dementia Assessment (MODA) (Brazzelli et al., 1994). This test is routinely used in our unit to evaluate patients for the presence of $\mathrm{MCl}$. The test is divided into three sections. The first section, "Orientation", assesses temporal, spatial, personal and family orientation. The second section, "Autonomy", investigates ADL. The third section, "Neuropsychological Testing", consists of nine brief

Table I - Demographic and clinical data of the study sample and the healthy controls.

\begin{tabular}{lll}
\hline & Healthy controls & $\mathrm{MCl}$ patients \\
\hline Males $(\mathrm{n})$ & 6 & 11 \\
Females $(\mathrm{n})$ & 4 & 2 \\
Mean age in years (SD) & $72(3.87)$ & $76(3.9)$ \\
Mean years of education (SD) & $11(4.45)$ & $6(3.1)$ \\
Mean MODA score (SD) & $93.91(4.49)$ & $84.67(7.4)$ \\
\hline
\end{tabular}

Abbreviations: $\mathrm{SD}=$ standard deviation; MODA=Milan Overall Dementia Assessment. 
tests that investigate attention, verbal intelligence, memory, verbal fluency, perception and identification of stimuli. The patients' verbal and metacognitive skills, knowledge of conversational rules, mood and other clinically relevant factors were also assessed by the neuropsychologist (AR) during the clinical evaluation. The control group was made up of healthy participants free from any clinical neurological or psychiatric conditions. The criteria for inclusion in the control group included absence of subjective cognitive complaints and absence of functional impairment. To further ascertain the absence of cognitive decline the healthy participants' cognitive status was evaluated using the MMSE (Folstein et al., 1975), which is the most frequently applied short cognitive test used to screen for and identify cognitive impairment. The MMSE is widely used in neuropsychological practice and consists of thirty items that assess orientation, short- and long-term memory, language, attention, visuospatial skills and the ability to follow simple verbal and written commands. This easy-to-use and relatively quick neuropsychological test is often employed to assess the overall cognitive status of elderly healthy people who serve as controls. Only one out of 10 participants had a score of 24 (cutoff score $23 / 24$ ), while all the others had scores ranging from 28 to 30 (two had a score of 28 , four had a score of 29 , and three had a score of 30 ). The clinical neuropsychologist, after further scrutiny of the MMSE performance by the participant who scored 24, approved his inclusion in the control group. In order to be able to compare the MODA scores obtained from the patients with the MMSE scores obtained from the healthy individuals, we used a formula proposed by Cazzaniga et al. (2003): MODA scores $=2.53 \mathrm{x}$ MMSE scores +21.55 . Participants were given a detailed explanation of the procedure and signed a written informed consent document approved by the Ethics Committee of Turin University.

\section{Procedures}

In the single-task condition (ST), the participants either walked or performed one of the three proposed cognitive tasks while seated. In the DT condition, they walked while carrying out one of the cognitive tasks.

The three cognitive tasks were: phonemic fluency (PF), short story recall (SS) and counting backwards (CB) by ones. Each cognitive performance was recorded and evaluated offline. The PF task consisted of the production of words beginning with the letters $F$, $A$ and $S$, one at a time, for 60 seconds (Spinnler and Tognoni, 1987). The number of correct responses was recorded. This task, in addition to requiring knowledge of lexical items and the ability to search semantic memory using phonological rules, also demands use of the executive functions involved in tracking prior responses and blocking intrusions. Hence, the PF task is a measure of processing efficiency and executive function. The SS task is one of the most reliable neuropsychological tests for evaluating verbal memory function (Della Sala, 1989). The participants were asked to listen to a short story, which was read by the experimenter, and immediately recall elements of the story. The number of correctly recalled items was recorded. Two short stories were used, both taken from the Italian version of the Rivermead Behavioral Memory Test (Della Sala, 1989). We employed the PF and SS tasks because they are commonly used to evaluate the presence of $\mathrm{MCl}$ in elderly people and therefore considered sensitive to differentiate these patients' performances from those of healthy controls. The CB task measures working memory processing, which is impaired early in the course of the disease. In this task the participants were asked to count backwards by ones starting either from 378 or 283 . The number of correctly produced digits was recorded. The CB task has previously been used to explore DT during walking in $\mathrm{MCl}$ patients (Montero-Odasso et al., 2009; Gillain et al., 2009) and has been found to perturb gait more than a verbal fluency task in older adults (Beauchet et al., 2005b). However this task is not generally used to assess the presence of cognitive decline in patients with a possible diagnosis of $\mathrm{MCl}$. In addition, the effect of a CB task depends greatly on the difficulty of the mental subtraction, i.e. counting backwards by sevens is much more difficult than counting backwards by ones (Muir et al., 2012). The gait task, performed in a well-lit gait laboratory, consisted of walking back and forth over a distance of 12 meters without stopping, at a self-selected pace. A gait analysis system (STEP 32, DEM Italia, Leinì, Turin, Italy) was employed, using three footswitches (sensors) placed on the sole of each bare foot (first and fifth metatarsal heads and the posterior part of the heel). Gait phases were calculated considering heel, sole and forefoot contact. The footswitch closing strength was $3 \mathrm{~N}$ and a sampling rate of $2 \mathrm{KHz}$ was used. A mean of 27 matching gait cycles was derived per condition. Acquired data were statistically processed offline by the system software and atypical gait cycles (i.e. with gait turns or acceleration and deceleration phases) were automatically excluded from the analysis. The assessed gait parameters were: stride time (StT), coefficient of variation of StT (CoV), calculated as the ratio between the standard deviation and the mean StT (CoV = [SD / mean StT] x 100), and the double support phase (DS), calculated as a percentage of gait cycle duration. Walking speed (WS) was also measured using a stopwatch. The mean value of two measures of WS over a distance of eight meters was considered. CoV expresses gait variability and is a measure of limb coordination and the risk of falls (Montero-Odasso et al., 2009, 2012). High CoV values have been related to impaired executive function (Allali et al., 2007). WS is a measure of gait stability and the risk of falls (Abellan van Kan et al., 2009) and has previously been used as a functional measure to assess the presence of $\mathrm{MCl}$ (Eggermont et al., 2010; Pettersson et al., 2007). Finally, higher DS values have been related to unsteadiness (Benedetti et al., 2012). All the selected parameters have been shown to be possibly affected by DT conditions. 
The participants did not receive any instructions as regards the priority to be given to one task over the other. Each subject was tested in seven conditions: i) Gait ST; ii) PF ST, iii) SS ST, iv) CB ST, v) PF DT; vi) SS DT, vii) CB DT. Each test lasted one minute and the order of the conditions was randomized across the participants.

\section{Statistical analysis}

Each gait parameter was analyzed using repeated measures analysis of variance (ANOVA) with Group (Patients vs Controls) as the between-subjects factor and Condition (ST, DT/PF, DT/SS, DT/BC) as the within-subjects factor. The participants' cognitive performance on each task was also analyzed using repeated measures ANOVA with Group (Patients vs Controls) as the between-subjects factor and Condition (ST, DT) as the within-subjects factor. Post-hoc analyses were performed using Duncan's test.

\section{Results}

The participants' demographic and clinical data are reported in table I. Age and educational level did not significantly differ between groups according to the results of repeated-measures t-tests. The MODA scores were significantly different between the two groups. As expected the $\mathrm{MCl}$ group had a significantly lower mean score than the control group [t-test $(21)=3.46 \mathrm{p}=0.002]$.

\section{Motor performance}

The gait data for the ST and the DT conditions are presented in table II. For the parameter WS, significant effects of Group $\left[F(1,21)=4.28, p=0.05, \eta_{p}{ }^{2}=\right.$ .169] and Condition $\left[F(3,63)=29.98, p<0.0001, \eta_{p}^{2}=\right.$ .588] were observed. The interaction Group $x$ Condition was not significant $\left(\eta_{p}^{2}=.012\right)$. The mean WS was higher in the control group $(0.83 \pm 0.12)$ than in the patients $(0.67 \pm 0.25)$. In addition, for the factor Condition, post-hoc analyses showed a significant dif- ference $(p<0.01)$ between ST $(0.89 \pm 0.20)$ and each DT condition $(\mathrm{DT} / \mathrm{PF}=0.68 \pm 0.23$; DT/SS $=0.66 \pm 0.20$; $\mathrm{DT} / \mathrm{CB}=0.72 \pm 0.22$ ) and no significant differences between the DT conditions. In particular, the WS was higher during single tasking than during dual tasking, independently of the concomitant cognitive task. Similarly, for dual tasking, significant effects of Group $\left[F(1,21)=4.74, p=0.04, \eta_{p}^{2}=.184\right]$ and Condition $\left[F(3,63)=9.75, p<0.0001, \eta_{p}^{2}=.317\right]$ were found. The interaction Group x Condition was not significant $\left(\eta_{p}^{2}=\right.$ .057). The mean DS value was lower in the control group $(25.08 \pm 0.36)$ than in the patients $(30.88 \pm 0.55)$. In addition, for the factor Condition, post-hoc analyses showed a significant difference between ST $(25.57 \pm 6.92)$ and each DT $(p<0.01)$ condition (DT/PF $=29.48 \pm 7.86 ;$ DT/SS $=29.66 \pm 7.50 ; \mathrm{DT} / \mathrm{CB}=$ $28.72 \pm 6.74$ ) but no significant differences between the DT conditions. In particular, DS was lower during ST than during each DT condition, independently of the cognitive task. For the other two gait parameters, StT and $\mathrm{CoV}$, the statistical analysis revealed a significant effect only for the factor Condition: StT $[F(3,63)=$ 10.65, $\left.p<0.0001, \eta_{p}^{2}=.336\right]$ and $\operatorname{CoV}[F(3,63)=6.74$, $\mathrm{p}<0.001, \eta_{p}^{2}=.243$ ]. For StT, post-hoc analyses showed a significant difference $(p<0.01)$ between ST $(1.18 \pm 0.21)$ and each DT condition (DT/PF = $1.32 \pm 0.30 ; \mathrm{DT} / \mathrm{SS}=1.32 \pm 0.25 ; \mathrm{DT} / \mathrm{CB}=1.28 \pm 0.25$ ), and no significant differences between the DT conditions. Similarly, for CoV, post-hoc analyses showed a significant difference $(p<0.01)$ between ST $(3.35 \pm 1.54)$ and each DT condition (DT/PF = $5.05 \pm 2.55 ; \mathrm{DT} / \mathrm{SS}=5.49 \pm 2.13 ; \mathrm{DT} / \mathrm{CB}=4.57 \pm 2.73$ ), but no significant differences between the DT conditions. Both StT and CoV increased from the ST to the DT conditions. The factor Group was not found to be significant for either StT $\left(\eta_{p}^{2}=.029\right)$ or $\operatorname{CoV}\left(\eta_{p}^{2}=\right.$ $.014)$ and no significant interactions were found between Group and Condition for either StT $\left(\eta_{p}^{2}=\right.$ $.018)$ or $\operatorname{CoV}\left(\eta_{p}^{2}=.06\right)$.

\section{Cognitive performance}

The data obtained in the ST and DT conditions are presented in table III. For the CB task, the statistical analyses showed a significant result only for the factor

Table II - Mean scores (SD) for each gait parameter in the study sample and the healthy controls.

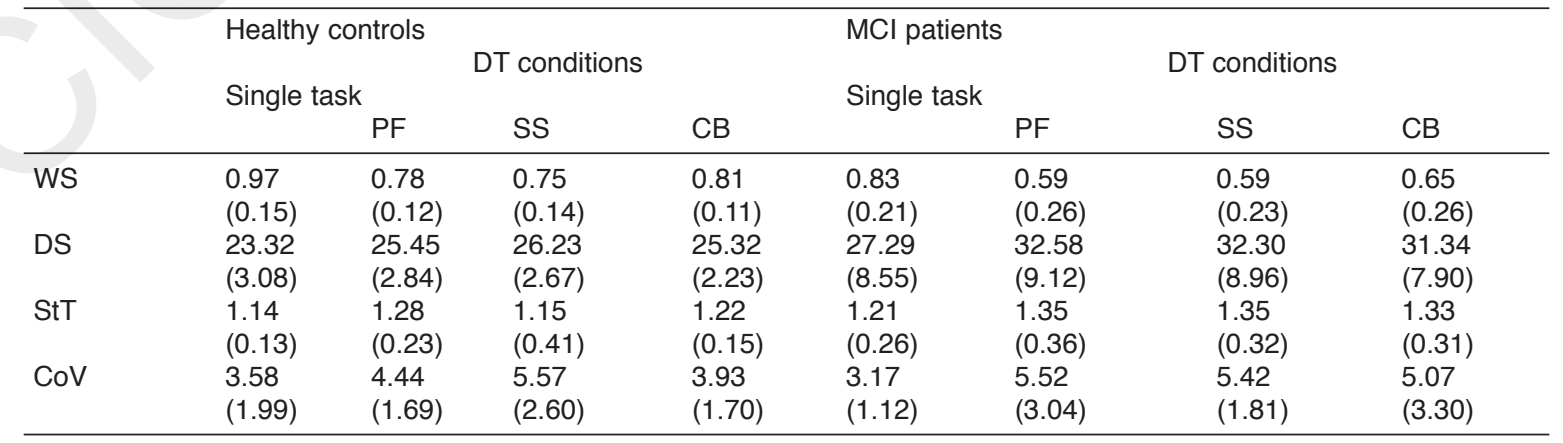

Abbreviations: $\mathrm{MCl}=$ mild cognitive impairment; $\mathrm{DT}=$ dual tasking; $\mathrm{PF}=$ phonemic fluency; $\mathrm{SS}=$ short story recall; $\mathrm{CB}=\mathrm{counting}$ backwards; $\mathrm{WS}=$ walking speed; DS=double support; $\mathrm{StT}=$ stride time; CoV=coefficient of variation of stride time 
Table III - Mean values (SD) for each cognitive task in the study sample and the healthy controls.

\begin{tabular}{lllll}
\hline & \multicolumn{2}{c}{ Healthy controls } & \multicolumn{2}{c}{ MCl patients } \\
& Single task & Dual task & Single task & Dual task \\
\hline Phonemic fluency & $10.40(4.45)$ & $8.10(4.07)$ & $4.76(4.23)$ & $4.23(2.89)$ \\
Short story recall & $5.85(3.22)$ & $7.70(3.21)$ & $2.50(2.26)$ & $2.88(2.87)$ \\
Counting backwards & $35.50(7.46)$ & $32.70(6.13)$ & $27.38(14.25)$ & $23.38(12.88)$ \\
\hline
\end{tabular}

Condition $\left[F(1,21)=5.4, p=0.03, \eta_{p}^{2}=.205\right]$. The factor Group $\left(p=0.065, \eta_{p}^{2}=.153\right)$ and the interaction Group $x$ Condition $\left(p=0.69, \eta_{p}^{2}=.008\right)$ were not significant. The members of both groups recorded a significantly higher number of correct digits under the ST $(31.44 \pm 12.26)$ than under the DT $(28.04 \pm 11.32)$ conditions. For the PF task, a significant difference was found only for the factor Group $\left(p=0.002, \eta_{p}^{2}=.363\right)$ : the number of correct words was significantly higher in the control group $(9.25 \pm 4.31)$ than in the group of patients $(4.5 \pm 3.56)$, independently of ST/DT conditions. The factor Condition $\left(p=0.132, \eta_{p}^{2}=.105\right)$ and the interaction Group $x$ Condition $\left(p=0.342, \eta_{p}^{2}=.043\right)$ were not significant. Finally, significant differences for both Group $\left[F(1,21)=13.74, p=0.001, \eta_{p}^{2}=.396\right]$ and Condition $\left[F(1,21)=5.08, p=0.04, \eta_{p}^{2}=.195\right]$ were found for the SS task. The interaction Group $x$ Condition was not significant $\left(p=0.154, \eta_{p}^{2}=.094\right)$. The number of correctly recalled items was significantly higher in the control group $(6.77 \pm 3.27)$ than in the group of patients $(2.69 \pm 2.54)$. In addition the number of correctly recalled items was significantly lower in the ST condition $(4.18 \pm 3.15)$ than in the DT condition $(5.29 \pm 3.83)$.

\section{Discussion}

Patients with $\mathrm{MCl}$ have some degree of gait impairment, and motor dysfunction has been shown to be related to the risk of developing Alzheimer's dementia (Aggarwal et al., 2006). Since DT conditions are a common aspect of everyday life and are also clinically relevant, as they carry a high risk of falls in the elderly population (Beauchet et al., 2009), previous studies have investigated their significance in $\mathrm{MCl}$ patients. Indeed, cognitive resources are more limited in $\mathrm{MCl}$ patients and might be overloaded during dual tasking. Thus, evidence that DT conditions significantly affect $\mathrm{MCl}$ patients with respect to healthy subjects might offer a rationale for the use of this paradigm as screening tool for the risk of developing dementia. However, previous studies exploring this possibility have given inconsistent findings (Pettersson et al., 2007; Maquet et al., 2010; Muir et al., 2012).

The results of our study support previous findings showing gait impairment (slower WS and higher DS) in $\mathrm{MCl}$ patients with respect to matched elderly controls, but they do not show the DT paradigm to be a sensitive tool for early $\mathrm{MCl}$ screening. Moreover, both groups showed an effect of DT interference on gait independently of the kind of concomitant cognitive task. An increase in gait variability during dual tasking may increase the risk of falls (Hausdorff et al., 2001).
Our results are consistent with the findings of Petterson et al. (2007), who reported lower WS in MCl patients with respect to controls during single tasking, but no differences between groups during dual tasking. Yet Pettersson et al. (2007) did not assess the effect of gait on cognitive performance. On the other hand, our results do not support previous findings showing a greater effect of DT interference on gait in $\mathrm{MCl}$ with respect to controls (Montero-Odasso et al., 2012; Maquet et al., 2010; Muir et al., 2012; Gillain et al., 2009). These inconsistent results may be explained by the different instruments used to assess gait parameters and the different cognitive tasks chosen, which may involve various degrees of attentional load. As predicted, the $\mathrm{MCl}$ patients performed worse than the controls on the SS and PF tasks, which mainly involve memory processes and executive functions, both of which are impaired early in the course of the disease. Moreover, reduced attentional resources may account for the worse performances on both these tasks. A main limitation of the present study is the use of the MMSE to assess the cognitive integrity of the control group, given the risk that the MMSE might have been less sensitive than the MODA (employed in the patient group) in detecting early signs of decline. However, given that we excluded subjects with MMSE scores just above the normal threshold who showed performances consistent with cognitive decline, and also the fact that the patients were found to be significantly impaired with respect to the controls in cognitive functions typically affected in $\mathrm{MCl}$ (SS and PF), this risk appears unlikely. Interestingly, the performances of the two groups did not differ on the CB task. This task may be too easy to detect early decline and therefore this result may reflect a ceiling effect. Alternatively, CB by ones may involve cognitive processes (i.e. working memory processing entailing a small memory load) that are still preserved in the early stages of $\mathrm{MCl}$. This second interpretation may fit better with the observation that this performance worsened during walking in both groups, indicating that cognitive resources were divided between gait and CB. As regards the PF task, neither group showed any effect of DT interference during walking, suggesting prioritization of the walking task, which requires executive functions. Unexpectedly, both groups showed enhanced performances on the SS task during gait. The finding that walking facilitates the immediate recall of a short story is in accordance with previous studies showing improved performance on memory and cognitive tasks soon after physical activities and exercise (Pontifex et al., 2009; Davranche and Audiffren, 2004). Thus, walking, rather than interfering with cognitive processing, may facili- 
tate the retrieval of information from episodic and/or semantic memory. This outcome might have important implications for the rehabilitation and prevention of memory deficits in $\mathrm{MCl}$ and for the planning of programs of cognitive stimulation and empowerment in both healthy and $\mathrm{MCl}$ individuals. To our knowledge, the SS task has never previously been employed in DT paradigms applied to $\mathrm{MCl}$ patients. The only enhancing effect (on both motor and cognitive tasks) previously found during dual tasking was observed when using $\mathrm{CB}$ by ones in elderly subjects with higher gait variability (Beauchet et al., 2010). However, CB by ones is a rhythmic and almost automatic task, and the enhanced gait performance in the study mentioned might be explained by a positive interaction with gait in subjects lacking rhythmicity. This is a very different condition from a story recall task in $\mathrm{MCl}$ patients. Further studies are needed to clarify the potential role of walking as a memory facilitator.

The lack of interactions between Group and Condition for both walking parameters and cognitive variables suggests that the DT condition affected our two groups' performances in a similar way. However, it is possible that because of the sample size and the heterogeneity of the $\mathrm{MCl}$ group, the statistical analyses were underpowered to reveal DT effects. Indeed, the small sample size constitutes another main limitation of the present study, even though previous works (Gillain et al., 2009; Maquet et al., 2010), with sample and group sizes similar to ours, have revealed greater effects of DT on gait in $\mathrm{MCl}$ patients with respect to controls. However, in our study, the effect sizes of the interactions were quite small (as indicated by the $\eta_{p}^{2}$ values) suggesting, from a clinical point of view, that dual tasking might not be a reliable paradigm for revealing early signs of cognitive decline in $\mathrm{MCl}$. On the other hand, our findings, consistently with the majority of previous DT studies in $\mathrm{MCl}$, strongly suggest the possibility that gait assessment per se might constitute a powerful and low-cost tool for revealing initial signs of cognitive impairment. Further research in larger groups of patients is necessary to clarify the impact of DT interference in this population.

Early gait disruption in $\mathrm{MCl}$ is clinically important as it entails a higher risk of falls and may therefore be an indication for preventive rehabilitation counselling. Overall, our $\mathrm{MCl}$ patients showed gait impairment in comparison to healthy controls. However, both groups manifested worse gait performance under DT than ST conditions, independently of the type of concomitant cognitive task. Likewise, in both groups, cognitive performance worsened on the CB task, did not change on the PF task, and unexpectedly improved on the SS task during dual tasking. The results of this study suggest that cognitive-gait interference during DT may not reveal small differences between $\mathrm{MCl}$ patients and healthy elderly controls and that the use of DT interference as an early screening tool is open to question. The unexpected finding of enhanced cognitive performance during walking might prompt further studies on beneficial effects of walking on cognitive functions in neurological patients and healthy subjects.

\section{References}

Abellan van Kan G, Rolland Y, Andrieu S, et al (2009). Gait speed at usual pace as a predictor of adverse outcomes in community-dwelling older people an International Academy on Nutrition and Aging (IANA) Task Force. J Nutr Health Aging 13:881-889.

Aggarwal NT, Wilson RS, Beck TL, et al (2006). Motor dysfunction in mild cognitive impairment and the risk of incident Alzheimer disease. Arch Neurol 63:1763-1769.

Allali G, Kressig RW, Assal F, et al (2007). Changes in gait while backcounting in demented older adults with frontal lobe dysfunction. Gait Posture 26:572-576.

Beauchet O, Dubost V, Herrmann FR, et al (2005a). Stride-tostride variability while backward counting among healthy young adults. J Neuroeng Rehabil 2:26.

Beauchet O, Annweiler C, Dubost V, et al (2009). Stops walking when talking: a predictor of falls in older adults? Eur $\mathrm{J}$ Neurol 16:786-795.

Beauchet O, Dubost V, Aminian K, et al (2005b). Dual-taskrelated gait changes in the elderly: does the type of cognitive task matter? J Mot Behav 37:259-264.

Beauchet O, Allali G, Poujol L, et al (2010). Decrease in gait variability while counting backward: a marker of "magnet effect"? J Neural Transm 117:1171-1176.

Benedetti MG, Agostini V, Knaflitz M, et al (2012). Self-reported gait unsteadiness in mildly impaired patients: an objective assessment through statistical gait analysis. J Neuroeng Rehabil 9:64.

Brazzelli M, Capitani E, Della Sala S, et al (1994). A neuropsychological instrument adding to the description of patients with suspected cortical dementia: the Milan overall dementia assessment. J Neurol Neurosurg Psychiatry 57:15101517.

Cazzaniga R, Francescani A, Saetti C, et al (2003). How to calculate an MMSE score from a MODA score (and vice versa) in patients with Alzheimer's disease. Neurol Sci 2003;24:261-267.

Davranche K, Audiffren M (2004). Facilitating effects of exercise on information processing. J Sports Sci 22:419-428.

Della Sala S (1989). Test di Memoria Comportamentale di Rivermead. Italian ed. Florence, Organizzazioni Speciali.

Dubost V, Annweiler C, Aminian K, et al (2008). Stride-to-stride variability while enumerating animal names among healthy young adults: result of stride velocity or effect of attentiondemanding task? Gait Posture 27:138-143

Dubost V, Kressig RW, Gonthier R, et al (2006). Relationships between dual-task related changes in stride velocity and stride time variability in healthy older adults. Hum Mov Sci 25:372-382.

Eggermont LH, Gavett BE, Volkers KM, et al (2010). Lowerextremity function in cognitively healthy aging, mild cognitive impairment, and Alzheimer's disease. Arch Phys Med Rehabil 91:584-588.

Folstein MF, Folstein SE, Mc Hugh PR (1975). "Mini-mental state". A practical method for grading the cognitive state of patients for the clinician. J Psychiatr Res 12:189-198.

Gauthier S, Reisberg B, Zaudig M, et al (2006). Mild cognitive impairment. Lancet 367:1262-1270.

Gillain S, Warzee E, Lekeu F, et al (2009). The value of instrumental gait analysis in elderly healthy, $\mathrm{MCl}$ or Alzheimer's disease subjects and a comparison with other clinical tests used in single and dual task conditions. Ann Phys Rehabil Med 52:453-474.

Hausdorff JM, Rios DA, Edelberg HK (2001). Gait variability and fall risk in community-living older adults: a 1-year prospective study. Arch Phys Med Rehabil 82:1050-1056. 
lavarone A, Ronga B, Pellegrino L, et al (2004). The Frontal Assessment Battery (FAB): normative data from an Italian sample and performances of patients with Alzheimer's disease and frontotemporal dementia. Funct Neurol 19:191-195.

Katz S, Ford AB, Moskowitz RW, et al (1963). Studies of illness in the aged. The index of ADL: a standardized measure of biological and psychosocial function. JAMA 185:914-919.

Lawton MP, Brody EM (1969). Assessment of older people: selfmaintaining and instrumental activities of daily living. Gerontologist 9:179-186.

Levinoff EJ, Saumier D, Chertkow H (2005). Focused attention deficits in patients with Alzheimer's disease and mild cognitive impairment. Brain Cogn 57:127-130.

Manckoundia P, Pfitzenmayer P, d'Athis P, et al (2006). Impact of cognitive task on the posture of elderly subjects with Alzheimer's disease compared to healthy elderly subjects. Mov Disord 21:236-241.

Maquet D, Lekeu F, Warzee E, et al (2010). Gait analysis in elderly adult patients with mild cognitive impairment and patients with mild Alzheimer's disease: simple versus dual task: a preliminary report. Clin Physiol Funct Imaging 30: 51-56.

Montero-Odasso M, Muir SW, Speechley M (2012). Dual-task complexity affects gait in people with mild cognitive impairment: the interplay between gait variability, dual tasking, and risk of falls. Arch Phys Med Rehabil 93:293-299.

Montero-Odasso M, Bergman H, Phillips NA, et al (2009). Dualtasking and gait in people with mild cognitive impairment. The effect of working memory. BMC Geriatr 9:41.
Muir SW, Speechley M, Wells J, et al (2012). Gait assessment in mild cognitive impairment and Alzheimer's disease: the effect of dual task challenges across the cognitive spectrum. Gait Posture 35:96-100.

Petersen RC, Negash S (2008). Mild cognitive impairment: an overview. CNS Spectr 13:45-53.

Pettersson AF, Olsson E, Wahlund LO (2007). Effect of divided attention on gait in subjects with and without cognitive impairment. J Geriatr Psychiatry Neurol 20:58-62.

Plummer-D'Amato P, Altmann LJ, Reilly K et al (2011). Dualtask effects of spontaneous speech and executive function on gait in aging: exaggerated effects in slow walkers. Gait Posture 33:233-237.

Pontifex MB, Hillman CH, Fernhall B, et al (2009). The effect of acute aerobic and resistance exercise on working memory. Med Sci Sports Exerc 41:927-934.

Sheridan PL, Hausdorff JM (2007). The role of higher-level cognitive function in gait: executive dysfunction contributes to fall risk in Alzheimer's disease. Dement Geriatr Cogn Disord 24:125-137.

Spinnler H, Tognoni G (1987). Standardizzazione e taratura italiana di test neuropsicologici. Ital J Neurol Sci 6 (Suppl 8).

Verghese J, Robbins M, Holtzer R, et al (2008). Gait dysfunction in mild cognitive impairment syndromes. J Am Geriatr Soc 56:1244-1251.

Woollacot M, Shumway-Cook A (2002). Attention and the control of posture and gait: a review of an emerging area of research. Gait Posture 16:1-14. 